\section{Untersuchung des Einflusses der Mitverbrennung von Abfäl- len in Zementwerken auf die Schwermetallbelastung des Produkts im Hinblick auf die Zulässigkeit der Abfallverwer- tung}

von M. Achternbosch, K.-R. Bräutigam, N. Hartlieb, Chr. Kupsch (ITAS); P. Stemmermann (ITC-WGT)*; U. Richers (ITC-ZTS)**

Im Auftrag des Umweltbundesamtes hat das Forschungszentrum Karlsruhe von November $\mathbf{2 0 0 0}$ bis März 2003 eine Studie zum Abfalleinsatz in Zementwerken durchgeführt. Ziel des Vorhabens war es, den Beitrag von Sekundärbrennstoffen, Sekundärrohstoffen und Zumahlstoffen zum Spurenelementgehalt von Zement und Beton darzustellen. Des Weiteren sollte untersucht werden, unter welchen Bedingungen und in welchem Maße die eingetragenen Spurenelemente in die Umwelt freigesetzt werden können. Zusätzlich sollte geprüft werden, welche Gesetze, Normen, Richtlinien usw. für die Produktion von Zement und den damit hergestellten Bauprodukten spezielle Anforderungen zu Schadstoffgehalten enthalten.

\section{Einführung}

Die Produktion von Industrie- und Konsumgütern ist mit einem hohen Verbrauch an Ressourcen verbunden. Nach ordnungsgemäßem Gebrauch fallen diese Güter als Abfall an. Bei der Gewinnung von Rohstoffen, der Herstellung, Nutzung und Entsorgung dieser Güter fallen zudem Abfälle und Abwässer an. Dies geschieht trotz des Wissens um die begrenzten stofflichen und energetischen Ressourcen der Erde und der Tatsache, dass die Umweltmedien nur in beschränktem Maße als Stoffsenke genutzt werden können. Aus diesem Grund sind Abfälle nach den Grundsätzen des Kreislaufwirtschafts- und Abfallgesetzes (KrW-/AbfG) in erster Linie zu vermeiden, insbesondere durch die Verminderung ihrer Menge und Schädlichkeit. Im Weiteren hat bei der Entsorgung eine Verwertung gegenüber der Beseitigung Vorrang. Es ist eine der Art und Beschaffenheit des Abfalls entsprechende hochwertige
Verwertung anzustreben. Die Verwertung kann stofflich oder energetisch erfolgen. Beide Verwertungsarten werden grundsätzlich als gleichrangig eingestuft. Es ist allerdings aufzuzeigen, von welcher Verwertungsart die geringsten Umweltbelastungen ausgehen.

Neben der Anforderung der Verwertung im $\mathrm{KrW}-/ \mathrm{AbfG}$ wird die Entsorgung von Abfällen durch andere Vorgaben der deutschen Abfallgesetze beeinflusst: Abfälle müssen vor ihrer $\mathrm{Ab}$ lagerung vorbehandelt werden. In Deutschland tritt diese Anforderung 2005 endgültig in Kraft, nach einer Übergangsfrist, in der es bisher nicht gelungen ist, die dafür notwendige Kapazität an Verbrennungsanlagen $\mathrm{zu}$ erstellen. Um den zu erwartenden Engpass zu beseitigen, wird derzeit propagiert, Abfälle zwecks Entsorgung in anderen thermischen Prozessen wie Kraftwerksfeuerungen, Zementdrehrohröfen und Industriefeuerungen einzusetzen.

Schon vor ca. 20 Jahren wurden heizwertreiche Fraktionen aus dem Hausmüll ausgeschleust und als Brennstoff aus Müll (BRAM) eingesetzt, der Regelbrennstoffe ersetzen sollte. Die Qualität von BRAM war aber mit der von Regelbrennstoffen nicht vergleichbar, so dass dieser Brennstoff an Bedeutung verlor. Die für die energetische Verwertung heute eingesetzten Abfälle laufen unter dem Schlagwort Sekundärbrennstoff. Sie zielen aber - mit verbesserten Methoden der Getrenntsammlung und Stofftrennung - in die gleiche Richtung: Schaffung von Entsorgungskapazität und Bereitstellung eines billigen, weil subventionierten Brennstoffs für die Industrie.

So gewinnen insbesondere die kostengünstigen Sekundärbrennstoffe bei der Zementherstellung aufgrund des hohen Anteils der Energiekosten an der Wertschöpfung des Produktes Zement immer mehr an Bedeutung. In den letzten Jahren ist der Abfalleinsatz in der Zementindustrie deutlich angestiegen. Der Anteil der Sekundärbrennstoffe an dem Gesamtbrennstoffeinsatz betrug 2002 etwa 35\%. Planungen erwägen einen weit höheren Einsatz. Da ein Großteil der Einsatzstoffe im Prinzip mehr oder weniger durch einen Sekundärstoff ersetzt werden kann, kann die Zementindustrie eine breite Palette an Alternativstoffen in Betracht ziehen.

Neben der Substitution primärer Brennstoffe kommen auch Abfälle als Sekundärrohstoffe und Zumahlstoffe zum Einsatz. 


\section{Problem- und Aufgabenstellung}

$\mathrm{Ob}$ es sich beim Einsatz von Abfällen als Sekundärbrennstoffe in der Zementindustrie im Sinne des KrW-/AbfG um eine ordnungsgemäße und schadlose Verwertung handelt, wird von Fachkreisen, Politik und Teilen der Gesellschaft zum Teil kontrovers diskutiert. Da die Abgasreinigungsanlagen der Zementwerke für den Produktionsprozess von Zement ausgelegt und nicht für eine Abfallverbrennung konzipiert sind, besteht die Sorge, dass der Abfalleinsatz möglicherweise zu erhöhten Emissionen von Spurenelementen führt.

Dies hat dazu geführt, dass neben der Diskussion um die Auswirkung des Abfalleinsatzes auf die Luftemission auch die Frage an Bedeutung gewinnt, in welchem Maße Spurenelemente aufgrund des Einsatzes von Abfällen in den Zement gelangen und ob daraus Umweltgefährdungen resultieren können. Dies betrifft neben den sekundären Brennstoffen auch die Verwendung von sekundären Rohstoffen als Rohmehlersatz, als Zumahlstoff für die Zementherstellung und als Zuschlagstoff bei der Herstellung von Beton.

Ziel des Vorhabens war es, den Einfluss unterschiedlicher Einsatzstoffe, insbesondere von Sekundärbrennstoffen, Sekundärrohstoffen, Zumahlstoffen und Betonzuschlägen auf den Spurenelementgehalt von Zement und Beton darzustellen. Von entscheidender Bedeutung für die Schadlosigkeit des Abfalleinsatzes ist die Frage, ob die Spurenelemente langfristig im Produkt, in diesem Falle im Beton, eingebunden sind und damit keine Gefährdung für die Umwelt entsteht. Aus diesem Grunde wurde der Kenntnisstand zum Bindungs- und Freisetzungsverhalten von Spurenelementen in Klinker, Zement und Beton dargestellt und Kenntnislücken wurden aufgezeigt.

Dieses im Auftrag des Umweltbundesamtes durchgeführte Vorhaben (Förderkennzeichen 20033 335) wurde von November 2000 bis März 2003 in Zusammenarbeit mit dem Institut für Technische Chemie durchgeführt, namentlich die Zentralabteilung Technikbedingte Stoffströme (ITC-ZTS) und der Bereich Wasser- und Geotechnologie (ITC-WGT).

\section{Vorgehensweise}

Der durchschnittliche Spurenelementgehalt des Zements und des Betons sowie der Beitrag einzelner Einsatzstoffe zu diesen Spurenelementgehalten wurden mit Hilfe von Stoffstromanalysen berechnet. Hierzu wurden Modellrechnungen auf Basis verschiedener Rechenansätze durchgeführt. Dabei kann davon ausgegangen werden, dass durch die Modellrechnungen die Auswirkungen des Einsatzes von Sekundärbrennstoffen auf den Spurenelementgehalt des Zements besser wiedergegeben werden können, als dies bei Messwerten der Fall ist, die lediglich eine Momentaufnahme darstellen und die von einer Reihe von Einflussfaktoren bestimmt werden.

Bei den mineralogischen Untersuchungen wurde der wissenschaftliche Kenntnisstand zum Bindungs-, Hydratations- und Freisetzungsverhalten von Spurenelementen in Zement und Beton aufgezeigt und bewertet.

\section{Ergebnisse}

Die Untersuchungen ergaben, dass die derzeit eingesetzten sekundären Einsatzstoffe einen geringen Anstieg der Konzentration einzelner Spurenelemente im Zement zur Folge haben. Eine Bewertung des Abfalleinsatzes bei der Zementherstellung und seiner Auswirkungen auf den Spurenelementeintrag kann jedoch nicht pauschal vorgenommen werden, sondern hängt von einer Reihe spezifischer Einflussfaktoren (betrachtetes Spurenelement, spezifisches Einsatzszenario der Abfälle etc) ab. Weiterhin lassen sich zukünftige Entwicklungen schwer abschätzen.

Die Freisetzung von Spurenelementen aus Betonbauteilen ist während der Nutzungsphase vernachlässigbar gering. Nach dem Abbruch ist ein erhöhter Spurenelementaustrag unter speziellen Annahmen denkbar. Der heutige Wissensstand reicht aber für eine abschließende Bewertung nicht aus.

Im Folgenden werden die Ergebnisse im Einzelnen aufgeführt:

(1) Ein Vergleich der errechneten durchschnittlichen Konzentrationen von Spurenelementen in Zementen mit entsprechenden Messwerten des Vereins Deutscher Zement 
werke e.V. (VDZ) zu Normalzementen zeigt eine gute Übereinstimmung.

(2) Sekundäre Einsatzstoffe tragen zum Spurenelementgehalt des Zements bei. Primäre Rohstoffe stellen zwar den wichtigsten Eintragspfad dar, sie sind allerdings nicht als die einzige wesentliche Quelle für den Spurenelementgehalt von Zement anzusehen. Neben dem Beitrag der Sekundärbrennstoffe spielen sekundäre Rohstoffe, insbesondere die Ersatzstoffe aus der Eisen- und Stahlindustrie sowie Steinkohlenflugaschen, eine wichtige Rolle. Wie groß der Anteil sekundärer Einsatzstoffe als Eintragspfad letztlich jedoch ausfällt, ist von Spurenelement zu Spurenelement unterschiedlich. Wie Abbildung 1 am Beispiel von Antimon und Blei zeigt, sind insbesondere Sekundärbrennstoffe, aber auch sekundäre Rohstoffe und hier insbesondere die Ersatzstoffe aus der Eisen- und Stahlindustrie sowie Steinkohlenflugaschen, von Bedeutung.

\section{Abb. 1: Anteil einzelner Eintragspfade am Gesamtgehalt von Antimon und Blei im Portlandzement}

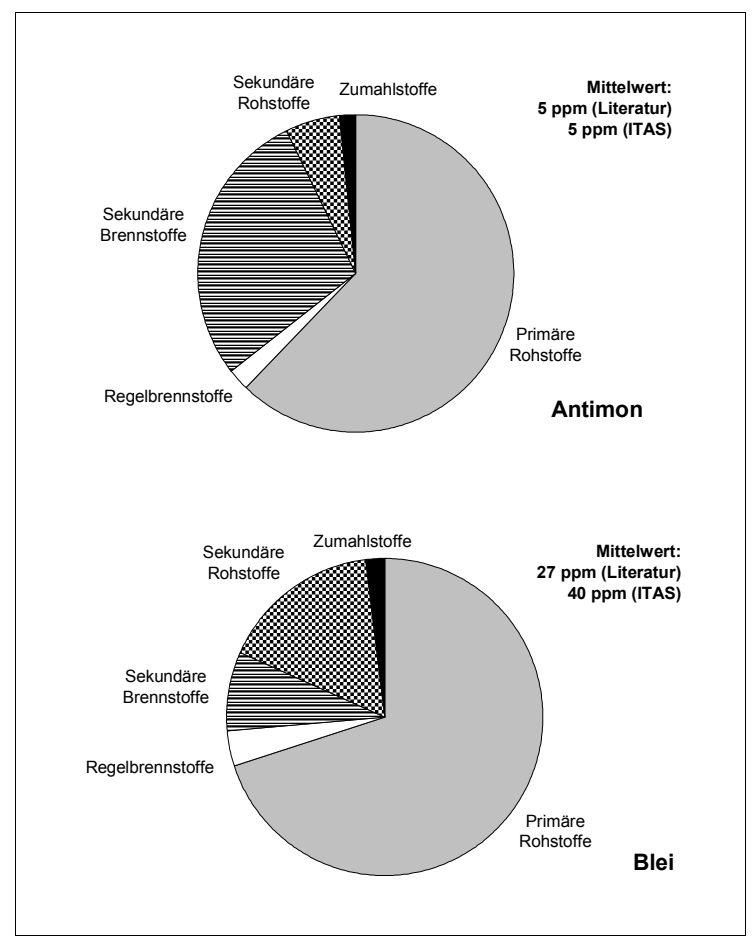

(3) Der verstärkte Einsatz von Abfällen im Jahr 2001 gegenüber 1999 führt rechnerisch zu einer leichten Zunahme im Spurenelementgehalt der Zemente. Für Cadmium, Antimon, Thallium und Zink ist eine Zunahme von bis zu $12 \% \mathrm{zu}$ beobachten. Für die anderen betrachteten Spurenelemente beläuft sich die Zunahme auf wenige Prozent und liegt damit in der Schwankungsbreite der Spurenelementkonzentrationen der Zemente für 1999. Im Jahr 2001 wurden, anders als 1999, große Mengen an Tiermehl als Sekundärbrennstoff eingesetzt. Tiermehl weist im Vergleich $\mathrm{zu}$ anderen Sekundärbrennstoffen relativ geringe Gehalte an Spurenelementen auf. Wären 2001 an Stelle von Tiermehl andere spurenelementreichere Sekundärbrennstoffe wie beispielsweise Industrie- und Gewerbeabfälle, Altreifen, Klärschlamm etc. eingesetzt worden, hätte dies zu einer deutlicheren Zunahme im Spurenelementgehalt der Zemente geführt.

(4) Der Einsatz von Abfällen bei der Zementherstellung zeigt für einzelne Spurenelemente Auswirkungen auf den Spurenelementgehalt von Zementen. Ein Anstieg der Konzentration im Zement aufgrund des Abfalleinsatzes lässt sich für Antimon, Cadmium und Zink mit der Berücksichtigung unterschiedlicher realistischer Szenarien eindeutig nachweisen. Die Szenarien wurden dabei so gewählt, dass der Zement in Szenario 1 einen möglichst geringen mittleren Spurenelementgehalt aufweist, indem nur natürliche Einsatzstoffe eingesetzt werden und auf Korrekturstoffe verzichtet wurde. Im Gegensatz dazu sind in Szenario 2 auch Korrekturstoffe berücksichtigt, eine in Deutschland typische Situation. Szenario 3 stellt einen „worst-case“-Fall dar, bei dem der aus dem Abfalleinsatz resultierende mittlere Spurenelementgehalt von Zement besonders groß ist. Abbildung 2 zeigt ausgewählte Ergebnisse dieser Berechnungen. In der Abbildung ist Cadmium exemplarisch für die Gruppe Antimon, Cadmium und Zink aufgeführt. 
Abb. 2: Mittelwerte der Spurenelementkonzentration und deren Bandbreiten im Portlandzement in den Szenarien 1 bis 3

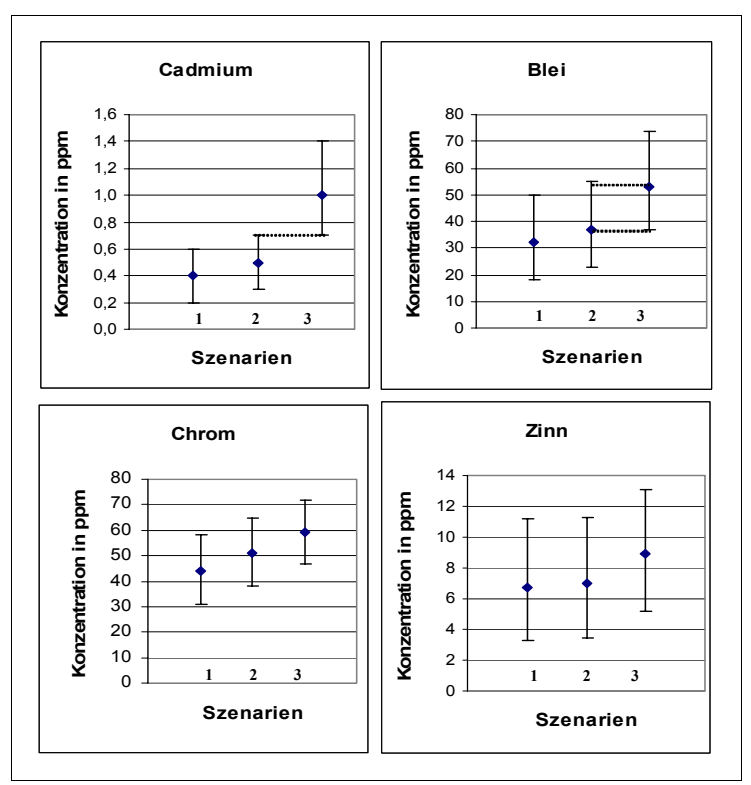

Für Blei und Kobalt (s. Beispiel Blei in der Abbildung) sind die Auswirkungen des Abfalleinsatzes nachweisbar, aber weniger deutlich. Für alle anderen untersuchten Spurenelemente zeigen sich in den Modellrechnungen keine signifikanten Effekte durch den Abfalleinsatz. Während für Antimon und Zink erhöhte Spurenelementgehalte des Zements auf den Einsatz von sekundären Brennstoffen zurückgeführt werden konnten, sind für Kobalt, Blei und Cadmium sekundäre Rohstoffe und Zumahlstoffe für einen leicht erhöhten Spurenelementeintrag in den Zement verantwortlich.

(5) Für den Spurenelementgehalt von Beton sind Zement, Steinkohlenflugasche und die $\mathrm{Zu}-$ schlagstoffe Basalt und Schlacke als Eintragspfade von Bedeutung. Eine Reduktion der Spurenelementkonzentration im Beton, insbesondere bei den Spurenelementen Kupfer, Nickel, Blei, Arsen und Zink, kann in den betrachteten Szenarien am ehesten dadurch erreicht werden, dass man auf den Einsatz von Steinkohlenflugasche als Zusatzstoff verzichtet. Entscheidend unter Umweltgesichtspunkten ist allerdings nicht der absolute Spurenelementgehalt von Beton, sondern der potenziell mobilisierbare Anteil. Unter physikalischen Gesichtspunkten können in Materialien mit einem großen Verhältnis Oberfläche zu Volumen (potenziell höhere Lösungskinetik) die darin enthaltenen Spurenelemente als potenziell langfristig mobilisierbar eingestuft werden. Danach ist nicht auszuschließen, dass Spurenelemente aus Bindemitteln wie Zementen und Steinkohlenflugaschen langfristig mobilisiert werden können.

(6) Alle in Zement aus unterschiedlichen Quellen eingetragenen Spurenelemente werden mittelfristig in Zementstein eingebunden. Dies gilt in gleicher Weise für Spurenelementfrachten aus Steinkohlenflugaschen, gleichgültig ob diese als Rohmehlersatz in der Zementherstellung, als Nebenbestandteil des Zements oder als Betonzusatzstoff verwendet werden.

(7) Für den Einsatz chlorhaltiger Sekundärstoffe werden zunehmend Gasbypässe zur Entlastung der Chloridkreisläufe im Zementbrennprozess installiert. Der anfallende Bypassstaub wird letztlich dem Zement zugemahlen. Durch diese Maßnahme wird es einerseits möglich, bisher nicht nutzbare chloridreiche Sekundärstoffe zeitlich begrenzt zur Produktion einzusetzen, da das kurzfristig eingebrachte Chlorid über einen langen Zeitraum dem Produkt zudosiert werden kann. Andererseits ist es möglich, den zulässigen Chloridgehalt im Produkt Zement weitestgehend auszuschöpfen. Damit können größere Mengen chloridreichere Sekundärstoffe als bisher eingesetzt werden. Der mittlere Chloridgehalt aller produzierten Zemente könnte sich bis auf den im Zement zulässigen Maximalwert erhöhen. Da die mit dem Einsatz chloridreicher Sekundärstoffe assoziierten Änderungen der Spurenelementfrachten nicht bekannt sind, lässt sich die zukünftige Entwicklung der Spurenelementgehalte von Zement schwer abschätzen. Die Ausschleusung des kleinen Stoffstroms aus Chlorbypässen könnte, falls erforderlich, für Minderungsmaßnahmen geeignet sein.

(8) Bereits wenige Stunden nach dem Anmachen von Frischbeton werden Spurenelemente nahezu vollständig im hoch alkalischen Bindemittel Zementstein fixiert. Das Bindemittel ist im Bereich pH 12-12,5 stark gepuffert. Solange der Puffer wirksam bleibt, bildet er für Spurenelemente eine stabile Senke. Dies gilt im Allgemeinen über den gesamten Nutzungszeitraum.

(9) Nach dem Abriss eines Bauwerks liegt Zementstein bzw. Beton in gebrochener Form vor. Betonbruch verwittert wegen der vergrö 
ßerten Oberfläche unter normalen Umweltbedingungen beschleunigt. Um ein Gefährdungspotenzial durch Spurenelemente zu vermeiden, ist die Art der Verwertung entscheidend. Wird die Oberfläche von Beton stark vergrößert, bricht die Pufferwirkung von Zementstein schnell zusammen. Die primär gebildeten Hydratphasen werden langsam umgewandelt, wobei die Mobilität von Spurenelementen verändert wird. Die zugrunde liegenden Reaktionen sind nur zum Teil bekannt und werden durch herkömmliche Testverfahren nicht abgebildet. Zur Vermeidung potenzieller Mobilisierungsmechanismen sollte eine Verwertung von Betonbruch räumlich konzentriert erfolgen, um das Pufferpotenzial des Zementsteins möglichst langfristig aufrecht $\mathrm{zu}$ erhalten. Im Idealfall sollte gebrochener Altbeton als Betonzuschlag und nicht im ungebundenen Straßenbau verwertet werden. Der Einfluss von Biota auf die Verwitterung nach der Nutzungsphase ist weitgehend unbekannt.

(10) Eine belastbare Prognose des langfristigen Spurenelementaustrags aus Betonbruch war in diesem Vorhaben aufgrund des derzeit vorhandenen wissenschaftlichen Kenntnisstandes nicht möglich. Ob nach der Nutzungsphase erhöht Spurenelemente freigesetzt werden, kann nicht eindeutig beantwortet werden. Die Einflussfaktoren (reaktive Oberflächen, $\mathrm{pH}$ des Milieus, Stabilität der Phasen und Verwitterungsprodukte etc.) sind äußerst komplex. Eine Prognose ist letztlich nur durch eine kombinierte Modellierung des Verwitterungsszenarios, des geochemischen Verhaltens der Zementphasen und der physikalischen Eigenschaften des Betons zu erreichen. Auch in näherer Zukunft werden solche Prognosen kaum möglich sein, da insbesondere zur geochemischen Modellierung zusätzlicher hoher Forschungsaufwand nötig ist. Dabei kann allerdings auf Vorarbeiten zur geochemischen Modellierung des Langzeitverhaltens von Zementstein in nuklearen Endlagern zurückgegriffen werden.

Eine englische Version des Projektabschlussberichts liegt in der Reihe Wissenschaftliche Berichte des Forschungszentrums Karlsruhe vor mit dem Titel „Heavy Metals in Cement and Concrete Resulting from the Co-Incineration of Wastes in Cement Kilns with Regard to the Legitimacy of Waste Utilisation“ (FZKA 6923) und ist auch auf der Homepage des Instituts als PDF-File verfügbar (http://bibliothek.fzk.de/zb/ berichte/FZKA6923.pdf).

* Institut für Technische Chemie, Bereich Wasserund Geotechnologie, Forschungszentrum Karlsruhe

** Institut für Technische Chemie, Zentralabteilung Technikbedingte Stoffströme, Forschungszentrum Karlsruhe

\section{Kontakt}

Forschungszentrum Karlsruhe in der HelmholtzGesellschaft GmbH

Institut für Technikfolgenabschätzung und Systemanalyse (ITAS)

Postfach 36 40, 76021 Karlsruhe

Fax: +49 (0) 7247 / 82 - 4806

Dr. Matthias Achternbosch

Tel.: +49 (0) 7247 / 82 - 4553

E-Mail: achternbosch@itas.fzk.de

Dipl.-Phys. Klaus-Rainer Bräutigam

Tel.: +49 (0) 7247 / 82 - 4873

E-Mail: braeutigam@itas.fzk.de

\section{$\langle\gg$}

